\title{
Electron Tomography Study on Junctions in Skin
}

\author{
Wanzhong He*, Pam Cowin**, David L. Stokes* \\ *Skirball Institute of Biomolecular Medicine $\quad * *$ Department of Cell Biology \\ New York University Medical Center, 540 First Avenue, New York, NY 10016
}

With recent progress in cellular electron tomography, it is feasible to obtain 5-8 $\mathrm{nm}$ resolution in three-dimensional structures from thin sections, thus revealing the structure and function of cellular subsystems ${ }^{[1-4]}$. Here we report our efforts for tomographic study of junctions in skin. There two types of junctions, adherens junctions and desmosomes (Fig.1), that couple cells together tightly and maintain the mechanical stability of tissues. This property is very important for skin and heart, which are subject to high shear forces. The purpose of this study is to reveal the organization and interactions between constituent proteins at about $5 \mathrm{~nm}$ resolution, and the structural mechanisms for assembling the junctions. We implemented our tomographic data collection system on our Philips CM200 FEG electron microscope, with Gatan 794 1kx1k slow-scan CCD controlled by DigitalMicrograph 2.5 running on Mac 8500 computer. Data collection is coordinated by the program EMACT running on an SGI workstation (Fig.2). EMACT was developed in David Agard and John Sedat (UCSF) groups, which relies on microscope communication program toolkit EMSCOPE developed in Bridget Carragher's group (Univ. of Illnios) and also on a plug-in program to Gatan's DigitalMicrograph. However, neither EMACT nor EMSCOPE functioned correctly with Philips control program version 12.5 before several bugs fixed and some communication addresses corrected. This software should now be useful for anyone having similar microscope and CCD. The image processing software we used for reconstruction are PRIISM (UCSF), IMOD (Univ. of Colorado), EM3D (Univ. of Stanford). The skin is taken from newborn mice, the skin samples are prepared in three ways: original skin, epidermis (dermis removed with dispase), and primary cell culture of keratinocytes. These samples are prepared with two different protocols, (1) chemical fixation with primary fixative $(3 \%$ glutaraldehyde, $0.2 \%$ tannic acid in MOPS buffer) and secondary fixative $\left(\% 1 \mathrm{OsO}_{4}\right)$, stained en bloc with $2 \%$ uranyl actetate, followed by progressive lowering of temperature dehydration with ethanol, and infiltrated and embedded with LX-112 resin; (2) cryofixation (high-pressure freezing or plunging into ethane), freeze substitution either in $1 \% \mathrm{OsO}_{4}, 0.1 \%$ uranyl actetate in acetone or in $0.2 \%$ Glutaraldehyde, $0.1 \%$ uranyl actetate in acetone, then infiltrated and embedded with LX-112 resin or LR white resin. Finally, 70-100nm sections are cut, stained with SATO lead stain solution, deposited with some $10 \mathrm{~nm}$ gold particles, coated with about $50 \mathrm{~nm}$ of amorphous carbon. In Fig. 3(a) a lot of junctions (white arrows) can be found between cells in the epidermis of chemically fixed skin. Fig. 3(b) is a chemically fixed cell culture sample, arrowheads point to desmosomes. Fig. 3(c) is an untilted projection image (taken at UCSF) of a desmosome linking two cultured keratinocytes. Fig. $3(\mathrm{~d})$, (e) are sections corresponding to the middle of the bottom of the reconstructed volume. We are currently focusing on specimen preparation in order to improve the resolution of this structure.

\section{References}

[1] A.J. Koster,H. Chen,J.W., Cedat, D. A. Agard.(1992),Ultramicroscopy,46:207-227.

[2] W. Baumeister, R. Grimm, J. Walz (1999), Trends Cell Biol.,9:81-85.

[3] J. R. McIntosh (2001), J. Cell Biol.,153:F25-F32.

[4] K. McDonald, M.K. Morphew(1993), Microscopy. Research. And Technique,24:465-473.

[5] P. Cowin, B. Burke(1996), Curr. Op. Cell Biol., 8:56-65. 

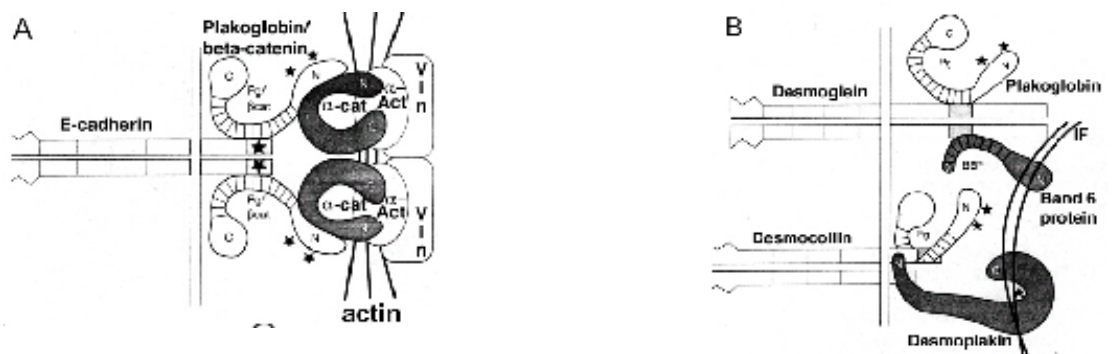

Fig.1 cartoon models of adherens junction $(\mathrm{A})$ and desmosomes $(\mathrm{B})^{[5]}$

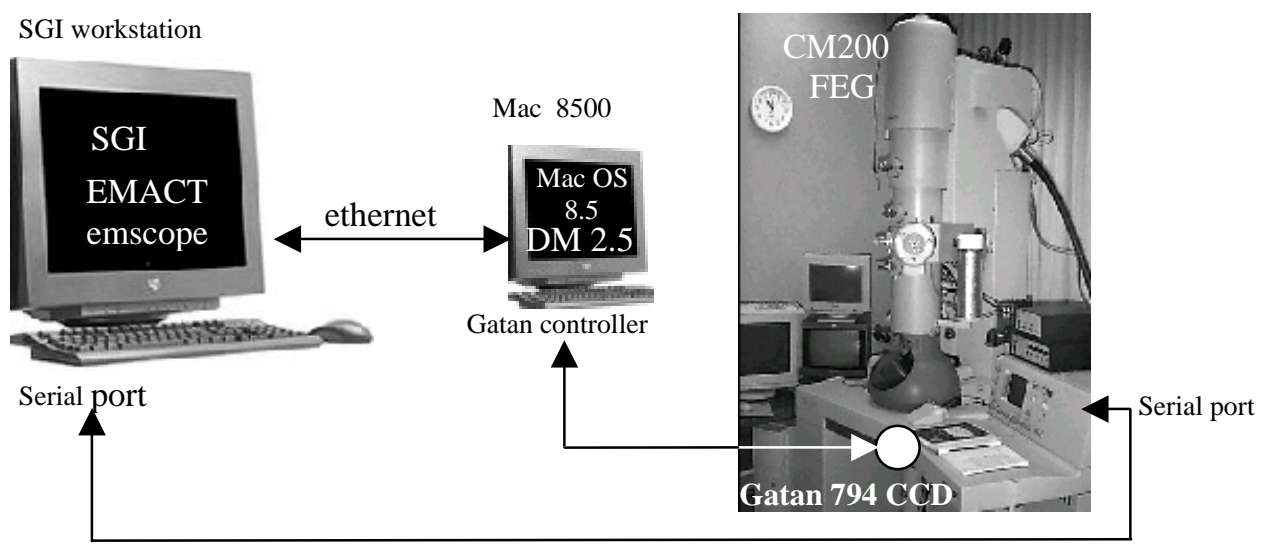

Fig.2 tomogram data collecting system
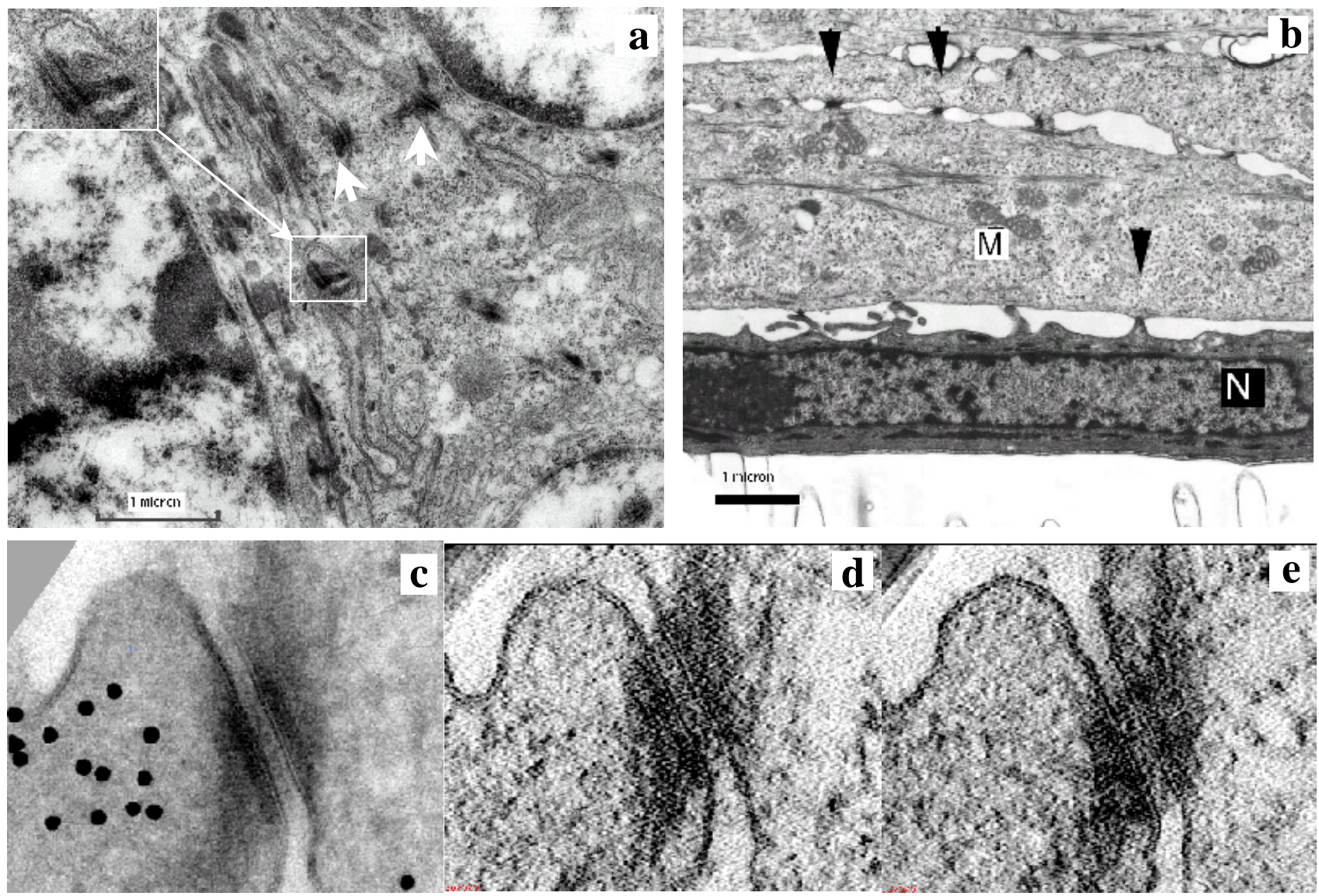

Fig.3 (a) junctions in epidermis (b) Junctions in cell culture (c) untilt tomgram (d) (e) reconstructed sections 